\title{
Production of Oxidants by Ion Bombardment of Icy Moons in the Outer Solar System
}

\author{
Philippe Boduch, ${ }^{1}$ Enio Frota da Silveira, ${ }^{2}$ Alicja Domaracka, ${ }^{1}$ Oscar Gomis, ${ }^{3}$ \\ Xue Yang Lv, ${ }^{1}$ Maria Elisabetta Palumbo, ${ }^{4}$ Sergio Pilling, ${ }^{5}$ Hermann Rothard, ${ }^{1}$ \\ Eduardo Seperuelo Duarte, ${ }^{6}$ and Giovanni Strazzulla ${ }^{4}$ \\ ${ }^{1}$ Centre de Recherche sur les Ions, les Matériaux et la Photonique (CIMAP), CIRIL-GANIL (CEA/CNRS/ENSI CAEN) \\ Université de Caen Basse-Normandie, 14076 Caen, France \\ ${ }^{2}$ Departamento de Física, Pontifícia Universidade Católica do Rio de Janeiro, 22451-900 Gávea, RJ, Brazil \\ ${ }^{3}$ Centro de Tecnologías Físicas, Universitat Politècnica de València, 46022 Valencia, Spain \\ ${ }^{4}$ INAF-Osservatorio Astrofisico di Catania, 95123 Catania, Italy \\ ${ }^{5}$ IP\&D, UNIVAP, 12299-00001 São Jose dos Campos, SP, Brazil \\ ${ }^{6}$ Grupo de Física e Astronomia, CEFET/Química de Nilópolis, 2653-060 Nilópolis, RJ, Brazil
}

Correspondence should be addressed to Giovanni Strazzulla, gianni@oact.inaf.it

Received 10 October 2011; Accepted 13 December 2011

Academic Editor: Francois Dulieu

Copyright (C 2011 Philippe Boduch et al. This is an open access article distributed under the Creative Commons Attribution License, which permits unrestricted use, distribution, and reproduction in any medium, provided the original work is properly cited.

Our groups in Brazil, France and Italy have been active, among others in the world, in performing experiments on physicalchemical effects induced by fast ions colliding with solids (frozen gases, carbonaceous and organic materials, silicates, etc.) of astrophysical interest. The used ions span a very large range of energies, from a few $\mathrm{keV}$ to hundreds $\mathrm{MeV}$. Here we present a summary of the results obtained so far on the formation of oxidants (hydrogen peroxide and ozone) after ion irradiation of frozen water, carbon dioxide and their mixtures. Irradiation of pure water ice produces hydrogen peroxide whatever is the used ion and at different temperatures. Irradiation of carbon dioxide and water frozen mixtures result in the production of molecules among which hydrogen peroxide and ozone. The experimental results are discussed in the light of the relevance they have to support the presence of an energy source for biosphere on Europa and other icy moons in the outer Solar System.

\section{Introduction}

Icy surfaces of the moons of planets in the outer Solar System [1] are exposed to different energetic processes among which irradiation by a number of energetic particle populations plays an important role. Cosmic rays, magnetospheric ions, solar flares, and solar wind particles continually bombard those surfaces producing many relevant and observed effects as simulated in some extent in laboratory experiments. As an example the observed presence of hydrogen peroxide $\left(\mathrm{H}_{2} \mathrm{O}_{2}\right)$ on Europa [2], Ganimede and Callisto [3] and ozone $\left(\mathrm{O}_{3}\right)$ for example, on Ganymede [4], Rhea and Dione [5] are commonly interpreted as due to the effects of irradiation by fast ion bombardment [6].
Although a large number of experiments have been performed in many laboratories in the world we are still far from having a complete picture of the many complex effects produced in space by the concurrent presence of several ion populations having, for example, a very large energy distribution and different charge state. Solar wind ions (fully ionized and with energies $\sim 1 \mathrm{keV} / \mathrm{u}$ ), multicharged magnetospheric ions (keV-MeV), and cosmic ions (energies up to $\mathrm{GeV}$ and more) all together irradiate the icy surfaces. It is then extremely important to compare the results obtained in different laboratories using ions having different energies with the aim to find the physical mechanisms that govern a given effect and be then able to confidently apply the results to astrophysical scenarios. An example of a successful result is 
the one relative to the sputtering of ices. Since the Publication of work by Brown et al. [7], it has been demonstrated that the sputtering yields of ices scale as the square of the energy lost by ions through electronic (inelastic) collisions (ionizations and excitations) with the atoms/molecules in the target $[8,9]$. This has been based on experiments made by ions having energies between few $\mathrm{keV}$ and some $\mathrm{MeV}$ and has been recently confirmed after experiments done with 100 's $\mathrm{MeV}$ ions on frozen $\mathrm{CO}_{2}$ and $\mathrm{CO}[10,11]$.

Our groups in Brazil, France, and Italy have been active, among others, in performing experiments in the field. The used ions span a very large range of energies, from a few $\mathrm{keV}$ to hundreds MeV.

Here we present a review and a comparison of the results obtained so far in our laboratories on the formation of oxidants (hydrogen peroxide and ozone) after ion irradiation of frozen water, carbon dioxide, and their mixtures. Irradiation of pure water ice produces hydrogen peroxide whatever is the used ion and at different temperatures (e.g., [12]). Irradiation of carbon dioxide and water frozen mixtures results in the production of hydrogen peroxide, ozone, and carbonic acid $[13,14]$. The experimental results will be discussed in the light of the relevance they have to explain the presence of oxidants that could also be an energy source for biosphere on Europa and other icy moons in the outer Solar System.

\section{Experimental}

The experiments here discussed were performed in two different apparatus at GANIL (Grand Accélérateur National d'Ions Lourds, Caen, France) and at LASp (Laboratorio di Astrofisica Sperimentale, INAF-Osservatorio Astrofisico di Catania, Italy) but having some common characteristics.

Pure or mixed gases were deposited at low temperature $(12-150 \mathrm{~K})$ onto IR transparent substrates (CsI in Caen, silicon double polished crystal in Catania) in high-vacuum chambers $\left(P<10^{-7} \mathrm{mbar}\right)$. Both of the chambers are faced, through IR-transparent windows, to a FTIR spectrophotometer (Nicolet Magna 500 in Caen, Bruker Equinox 55 in Catania). In Caen the sample-cryostat system can be rotated and fixed at three different positions to allow: gas deposition, FTIR measurement, and perpendicular ion irradiation. Ions are accelerated at high total energies (10's-100's MeV) and high charge state $[11,14]$. In Catania the substrate plane is placed at a 45 degree angle to the IR beam and the ion beam so that spectra can easily be taken in situ, even during irradiation, without tilting the sample. Ion beams with energies up to $200 \mathrm{keV}$ ( $400 \mathrm{keV}$ for double ionization) are obtained by an ion implanter [15].

In all of the experiment here shown the thickness of the samples was lower than the penetration depth of the used ion and ion current density was maintained low enough to avoid a macroscopic heating of the samples. The spectra were taken with a resolution of $1 \mathrm{~cm}^{-1}$. A list of the experiments whose results are here discussed is given in Table 1. Ions interact with the target molecules by losing energy through nuclear (elastic) or electronic (inelastic) collisions. The energy lost is redistributed among the species present in the target and causes displacements of atoms from their lattice position and breaking of molecular bonds whose recombination may lead to the production of new species. The specific energy loss or stopping power (energy lost per unit of path length of the ion in the target) has been calculated using the SRIM [16] software and is reported in Table 1 for the inelastic (electronic stopping, Se) components. For all of the considered ions the elastic (nuclear stopping) is negligible and for all of the targets a density of $1 \mathrm{~g} / \mathrm{cm}^{3}$ is assumed. The maximum total energy released to the sample by impinging ions (dose) is also reported in Table 1 and it is given in $\mathrm{eV} / 16 \mathrm{u}$, where $\mathrm{u}$ is the unified atomic mass unit defined as $1 / 12$ of the mass of an isolated atom of carbon-12. This is a convenient way to characterize chemical changes and compare the results obtained irradiating different samples [17].

In most cases, the column density $N$ (molecules $/ \mathrm{cm}^{2}$ ) of a species has been calculated by dividing the area $\left(\mathrm{cm}^{-1}\right)$ of a given band (measured in an optical depth scale) by its band strength $\left(\mathrm{cm}\right.$ molecule $\left.{ }^{-1}\right)$. The band area was calculated by removing the underlying continuum. In Table 2 , the peak positions of the band used to evaluate the column density of a given species are reported together with the used band strength. In some instances, the band of ${ }^{12} \mathrm{CO}_{2}$ at $2343 \mathrm{~cm}^{-1}$ was saturated, in which case the column density was calculated using the band of the isotope ${ }^{13} \mathrm{CO}_{2}$ at $2280 \mathrm{~cm}^{-1}$. We assumed that the values for the band strength of ${ }^{12} \mathrm{CO}_{2}$ and ${ }^{13} \mathrm{CO}_{2}$ are the same.

The band strength values given in Table 2 for water and carbon dioxide refer to pure molecules. For $\mathrm{H}_{2} \mathrm{O}_{2}$ it refers to a concentration of $2.66 \%$ by mass or $1.4 \%$ by number relative to $\mathrm{H}_{2} \mathrm{O}$ [20]. For $\mathrm{O}_{3}$ it is the value measured in the gas phase [21] that is usually used and it is assumed to be acceptable for the molecule diluted in a matrix. When we have deposited $\mathrm{CO}_{2}$ rich mixtures we have done calibration experiments such that the partial pressure of the two species in the mixing chamber corresponds to deposition rates adequate, within $10 \%$, to produce the wanted molecular ratio in the solid phase.

The transmittance of the sample is partly due to the transparence of the solid substrate in the IR range. Ion irradiation of the substrate may result in its structure modification, for example, in its amorphization as it can be the case for the silicon crystal, then a part of the relative transmittance evolution of the continuum could be associated with the substrate deterioration during the irradiation. However the area of the molecular bands has been obtained, in optical depth scale, after the subtraction of the underlying continuum and is then not affected from the "damage" that the substrate might have.

\section{Results}

3.1. The Fate of Deposited Molecules. The chemistry of irradiated pure water and carbon dioxide ices starts with the dissociation of $\mathrm{H}_{2} \mathrm{O} \rightarrow \mathrm{H}+\mathrm{OH}$ or $\mathrm{CO}_{2} \rightarrow \mathrm{O}+\mathrm{CO}$ followed by the formation of many neutral, excited, and 
TABLE 1: Summary of the experiments discussed in this paper.

\begin{tabular}{|c|c|c|c|c|c|}
\hline $\begin{array}{l}\text { Target } \\
\mathrm{H}_{2} \mathrm{O}: \mathrm{CO}_{2}\end{array}$ & Thickness $10^{17} \mathrm{~mol} / \mathrm{cm}^{2}$ & $T(\mathrm{~K})$ & Ion $(E$ in $\mathrm{MeV})$ & $\begin{array}{c}\text { Electronic stopping } \\
\text { power } \mathrm{e} / \AA^{-1}\end{array}$ & Max dose $(\mathrm{eV} / 16 \mathrm{u})$ \\
\hline $1: 0$ & 2.4 & 16 & $\mathrm{He}^{+}(0.2)$ & 16 & 186 \\
\hline $1: 0$ & 2.3 & 77 & $\mathrm{He}^{+}(0.2)$ & 16 & 23 \\
\hline $1: 0$ & 2.0 & 16 & $\mathrm{H}^{+}(0.2)$ & 7 & 180 \\
\hline $1: 0$ & 2.5 & 77 & $\mathrm{H}^{+}(0.2)$ & 7 & 40 \\
\hline $1: 0$ & 34 & 12 & ${ }^{58} \mathrm{Ni}^{11+}(46)$ & 490 & 30 \\
\hline $1: 0$ & 33 & 13 & ${ }^{129} \mathrm{Xe}^{23+}(92)$ & 849 & 23 \\
\hline $1: 0$ & 14 & 13 & ${ }^{58} \mathrm{Ni}^{13+}(52)$ & 492 & 13 \\
\hline $10: 1$ & 6.9 & 13 & ${ }^{58} \mathrm{Ni}^{13+}(52)$ & 466 & 5 \\
\hline $5: 1$ & 7.0 & 16 & $\mathrm{He}^{+}(0.2)$ & 15.1 & 75 \\
\hline $2.5: 1$ & 4.5 & 77 & $\mathrm{He}^{+}(0.2)$ & 14.5 & 23 \\
\hline $1: 1$ & 9.9 & 13 & ${ }^{58} \mathrm{Ni}^{13+}(52)$ & 398 & 11 \\
\hline $1: 1$ & 3.0 & 16 & $\mathrm{He}^{+}(0.2)$ & 13.8 & 22 \\
\hline $0: 1$ & 7.5 & 13 & ${ }^{58} \mathrm{Ni}^{13+}(52)$ & 668 & 12 \\
\hline
\end{tabular}

TABle 2: Peak positions $\left(\mathrm{cm}^{-1}\right)$ of the bands used to evaluate the column density of the given species and the band strengths $(\mathrm{cm}$ molecule $\left.{ }^{-1}\right)$.

\begin{tabular}{lccc}
\hline Molecule & $\begin{array}{c}\text { Peak position } \\
\text { cm }^{-1}\end{array}$ & $\begin{array}{c}\text { Band strengths } \\
\text { cm molecule }\end{array}$ & Reference \\
\hline $\mathrm{H}_{2} \mathrm{O}$ & 3300 & $2.0 \times 10^{-16}$ & {$[18]$} \\
$\mathrm{CO}_{2}$ & 2344 & $7.6 \times 10^{-17}$ & {$[19]$} \\
$\mathrm{H}_{2} \mathrm{O}_{2}$ & 2850 & $2.7 \times 10^{-17}$ & {$[20]$} \\
$\mathrm{O}_{3}$ & 1040 & $1.0 \times 10^{-17}$ & {$[21]$} \\
\hline
\end{tabular}

ionized species. The fragments have a chance to recombine and lead to the formation of species originally not present in the target, while the number of original molecules decreases progressively with the increase of the ion fluence. The most abundant species are $\mathrm{H}_{2} \mathrm{O}_{2}$ (i.e., formed also from pure water), $\mathrm{CO}, \mathrm{CO}_{3}, \mathrm{H}_{2} \mathrm{CO}_{3}$, and $\mathrm{O}_{3}$. Here we concentrate on the formation of the oxidants hydrogen peroxide and ozone. The "disappearance" of the original molecules is due to two different causes: sputtering (i.e., molecules or their fragments are ejected from the target) and chemical alteration (i.e., the fragments form species that reside on the target). The disappearance of original molecule is not stoichiometric that is, the column density ratio $\left(\mathrm{CO}_{2} / \mathrm{H}_{2} \mathrm{O}\right.$ in the present case) changes with ion fluence.

As exemplified in Figure 1, the column density ratio $\mathrm{CO}_{2} / \mathrm{H}_{2} \mathrm{O}$ decreases for all of the experiments we made on mixtures irrespective of the temperature of the target $(12-80 \mathrm{~K})$, of the starting mixture $\left(\mathrm{H}_{2} \mathrm{O} / \mathrm{CO}_{2}\right.$ from $1: 1$ to $10: 1)$ and ion energy that spans over several orders of magnitude. Such a decrease is partially due to the sputtering yield that is higher for carbon dioxide than for water ice (by a factor 10-20) but mostly to the fact that most of the fragments produced from water reform water molecules when recombine. On the contrary the first fragment of carbon dioxide is $\mathrm{CO}$ that is stable and hardly recombines into $\mathrm{CO}_{2}$. An equilibrium value at high fluences is expected

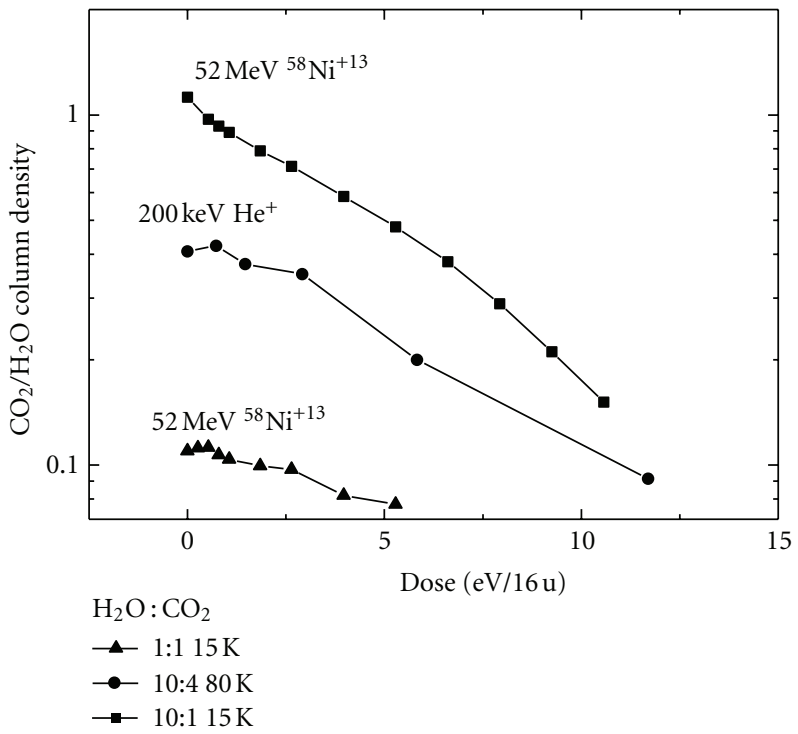

Figure 1: The column density ratio $\left(\mathrm{CO}_{2} / \mathrm{H}_{2} \mathrm{O}\right)$ of three different mixtures at 15 or $80 \mathrm{~K}$, is reported versus the dose suffered after irradiation with $\mathrm{He}^{+}$or $\mathrm{Ni}^{13+}$ ions having different energies.

when the number of carbon dioxide molecules that are destroyed balances the number of molecules formed by the other species formed (e.g., CO). This value is lower than $\mathrm{CO}_{2} / \mathrm{H}_{2} \mathrm{O}=0.08$ (Figure 1). Note that also for sputtering, an equilibrium value of the surface stoichiometry is expected being the absolute number of sputtered molecules proportional to their concentration in the target.

\subsection{Synthesis of Oxidant Species: $\mathrm{H}_{2} \mathrm{O}_{2}$}

3.2.1. In Water Ice. Hydrogen peroxide is formed after irradiation of pure water ice (e.g., $[12,20])$ and all of the irradiated mixtures both at low $(15 \mathrm{~K})$ and higher $(80 \mathrm{~K})$ temperature and whatever is the ion projectile, its energy, and charge state. As examples see the band at about $2850 \mathrm{~cm}^{-1}$ in Figures 2 


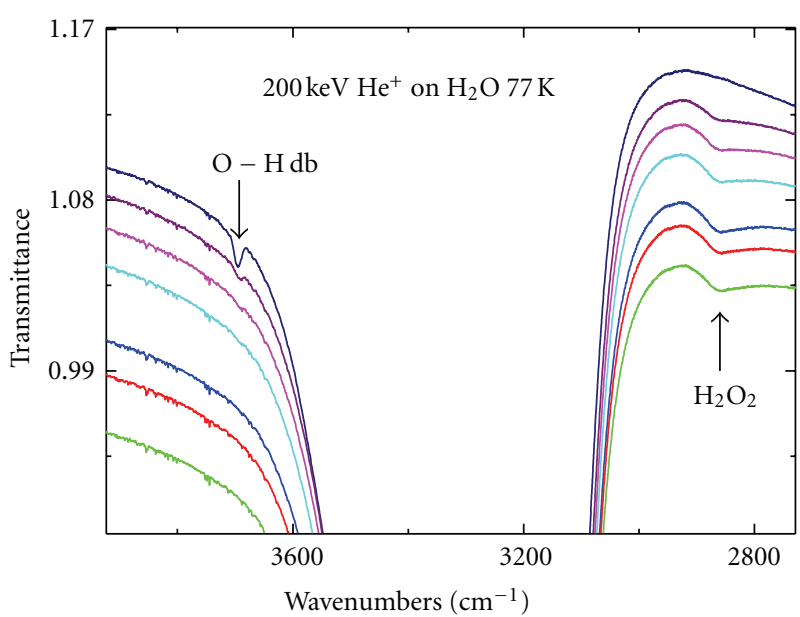

Flux

$10^{14}$ ions $\mathrm{cm}^{-2}$

$\begin{array}{lll}0 & -0.6 & - \\ - & & 2.4 \\ 0 & 1.2 & -5\end{array}$

$-0.4$

FIgure 2: IR spectra (shifted for clarity) of $\mathrm{H}_{2} \mathrm{O}$ ice as deposited and after irradiation with $200 \mathrm{keV} \mathrm{He} \mathrm{He}^{+}$at $77 \mathrm{~K}$. It is clear that the appearance, upon irradiation, of a band at about $2850 \mathrm{~cm}^{-1}$ is easily attributed to $\mathrm{H}_{2} \mathrm{O}_{2}$.

and 3. In Figure 2 we show the IR transmittance spectra of $\mathrm{H}_{2} \mathrm{O}$ ice as deposited and after irradiation with $200 \mathrm{keV} \mathrm{He}$ at $77 \mathrm{~K}$. In Figure 3 the IR spectra, in absorbance scale, of $\mathrm{H}_{2} \mathrm{O}$ ice as deposited and after irradiation with $52 \mathrm{MeV} \mathrm{Ni}^{+13}$ at $15 \mathrm{~K}$ are shown. In both cases it is clear the formation of the band at about $2850 \mathrm{~cm}^{-1}$ whose intensity increases with ion fluence and easily attributed to $\mathrm{H}_{2} \mathrm{O}_{2}$. From Figure 2 it is also clear the presence in the original ice of a band at about $3695 \mathrm{~cm}^{-1}$ that is due to the $\mathrm{O}-\mathrm{H}$ dangling bonds. This band is characteristic of a noncrystalline ice and it is due to the presence of pores. Its profile (shape, peak position, and FWHM) and intensity vary as a function of temperature and the species with which the water ice is mixed. A crystalline water ice does not exhibit this band. It has been already demonstrated that ion irradiation causes a "compaction" of the amorphous ice [22] with the consequent disappearance of that band as also evident for the data shown in Figure 2 obtained after irradiation at $77 \mathrm{~K}$.

The column density ratios $\mathrm{H}_{2} \mathrm{O}_{2} / \mathrm{H}_{2} \mathrm{O}$ obtained after irradiation of pure water ice $(13-16 \mathrm{~K})$ are displayed in Figure 4 (open and full triangles) versus the dose deposited by two different ions having different energies $\left(200 \mathrm{keV} \mathrm{He}^{+}\right.$ and $52 \mathrm{MeV} \mathrm{N}^{+13}$ ). We can see that in pure water ice the $\mathrm{H}_{2} \mathrm{O}_{2} / \mathrm{H}_{2} \mathrm{O}$ number ratio stabilizes to a value of $\sim 0.02$ at low $T$. At higher $T(70-100 \mathrm{~K})$, more appropriate for the icy moons, this value is lower of about a factor 2 [12]. The evolution of the number ratio with ion fluence (i.e., with the deposited dose) is due to a formation process that produces hydrogen peroxide from water and a destruction process of the formed peroxide.

3.2.2. In Water/Carbon Dioxide Icy Mixtures. The addition of $\mathrm{CO}_{2}$ to water ice causes an increase in the concentration

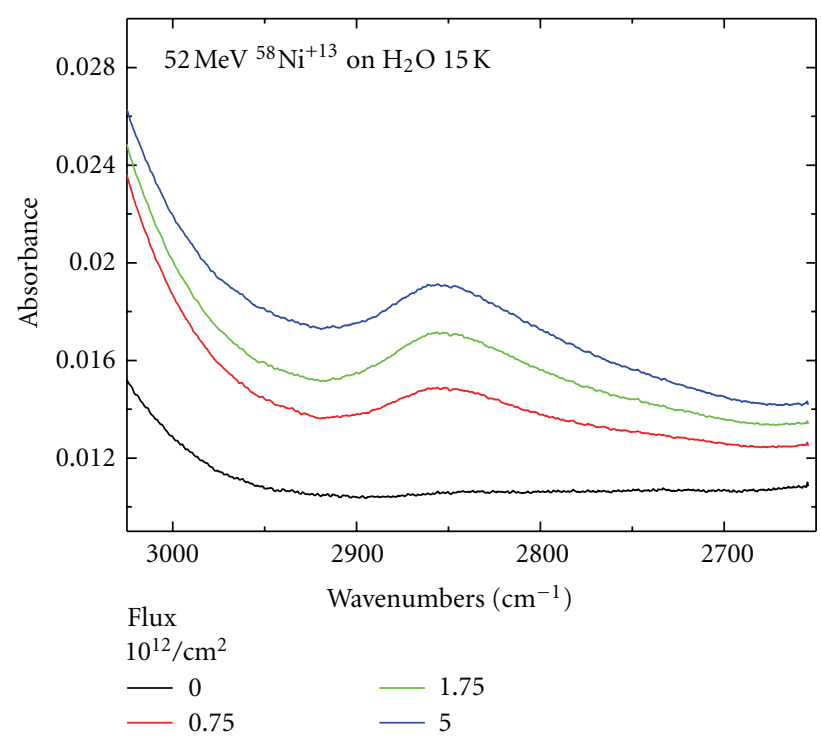

FIGURE 3: IR spectra (shifted for clarity) of $\mathrm{H}_{2} \mathrm{O}$ ice as deposited and after irradiation with $52 \mathrm{MeV} \mathrm{Ni} \mathrm{Ni}^{+13}$ at $15 \mathrm{~K}$. It is clear the appearance, upon irradiation, of a band at about $2850 \mathrm{~cm}^{-1}$ easily attributed to $\mathrm{H}_{2} \mathrm{O}_{2}$.

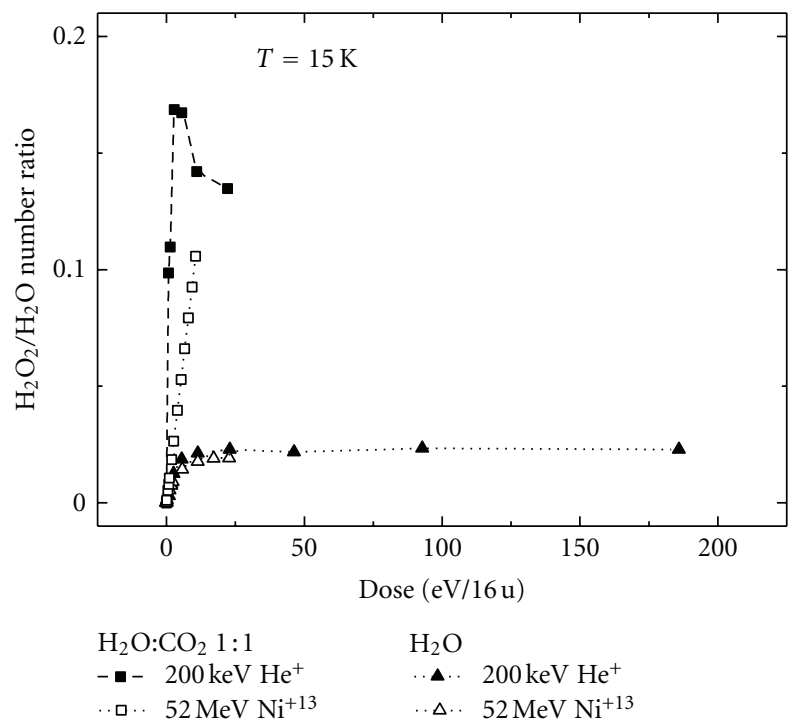

Figure 4: The column density ratios $\mathrm{H}_{2} \mathrm{O}_{2} / \mathrm{H}_{2} \mathrm{O}$ obtained after irradiation of pure water ice and a mixture $\mathrm{H}_{2} \mathrm{O}: \mathrm{CO}_{2}=1: 1$ are reported versus the dose deposited by two different ions having different energies.

of hydrogen peroxide as exemplified in Figure 4 where the column density ratio $\mathrm{H}_{2} \mathrm{O}_{2} / \mathrm{H}_{2} \mathrm{O}$ obtained after irradiation of a mixture $\mathrm{H}_{2} \mathrm{O}: \mathrm{CO}_{2}=1: 1$ is shown versus the dose deposited by two different ions having different energies.

Although other possibilities exist, it is usually believed [8, $20]$ that the chemistry of irradiated water/carbon dioxide ice mixtures start preferentially with the dissociations:

$$
\mathrm{H}_{2} \mathrm{O} \longrightarrow \mathrm{H}+\mathrm{OH}, \quad \mathrm{CO}_{2} \longrightarrow \mathrm{O}+\mathrm{CO}
$$


followed by the formation of many neutral, excited, and ionized species: $\mathrm{H}, \mathrm{H}_{2}, \mathrm{O}, \mathrm{O}_{2}, \mathrm{H}_{2} \mathrm{O}, \mathrm{HO}_{2}, \mathrm{H}_{2} \mathrm{O}_{2}, \mathrm{O}_{3}, \mathrm{HCO}$, $\mathrm{CO}_{3}, \mathrm{H}_{2} \mathrm{CO}_{3}$, and others (e.g., $\left.[8,23,24]\right)$. The initial radiation effects may be directly due to the inelastic collision of the ions with the molecules and/or to ionizations and excitations that produce ions and electrons which may either enter into chemical reactions or, given sufficient energy, form a separate track of radiation events.

It is important to note that the backreaction $\mathrm{H}+\mathrm{OH} \rightarrow$ $\mathrm{H}_{2} \mathrm{O}$ is highly favored and in fact most of the water fragments recombine to reform water when pure ice is irradiated. Viceversa, the backreaction $\mathrm{O}+\mathrm{CO} \rightarrow \mathrm{CO}_{2}$ is not favored and irradiated carbon dioxide evolves versus a mixture with carbon monoxide (up to $25 \%$ as discussed below, see Figure 6(b)) and other species (e.g., [13, 14]). This allows the $\mathrm{O}$ atoms (from $\mathrm{CO}_{2}$ ) to favor the formation of oxidant species. In the case of hydrogen peroxide the dissociations of (1) are followed by other reactions such as

$$
\mathrm{O}_{\left(\text {from } \mathrm{CO}_{2}\right)}+\mathrm{H}_{\left(\text {from } \mathrm{H}_{2} \mathrm{O}\right)} \longrightarrow \mathrm{OH}+\mathrm{OH} \longrightarrow \mathrm{H}_{2} \mathrm{O}_{2}
$$

As said above, the different behavior of water and carbon dioxide (along with the higher sputtering yield of $\mathrm{CO}_{2}$ ) explains not only why the column density ratio $\mathrm{CO}_{2} / \mathrm{H}_{2} \mathrm{O}$ decreases with increasing ion fluence but also why the amount of hydrogen peroxide produced after irradiation of mixtures reaches a maximum and then decreases when the amount of the carbon dioxide decreases. This is evidenced by the results presented in Figure 4 and relative to a mixture $\mathrm{H}_{2} \mathrm{O}: \mathrm{CO}_{2}=1: 1$ irradiated by $200 \mathrm{keV} \mathrm{He}^{+}$ions (see [12] for further evidences). We believe that this is not evident for the experiment done with high energy ions because we had to stop the experiment before the maximum value of the $\mathrm{H}_{2} \mathrm{O}_{2} / \mathrm{H}_{2} \mathrm{O}$ was obtained.

3.3. Synthesis of Oxidant Species: $\mathrm{O}_{3} \cdot \mathrm{O}_{3}$ was detected only after irradiation of pure $\mathrm{CO}_{2}$ and of the mixture $1: 1$, that is, with the largest amount of $\mathrm{CO}_{2}$ (Figure 5). Its column density increases with ion fluence, reaches a maximum $\left(\mathrm{O}_{3} / \mathrm{H}_{2} \mathrm{O} \sim 0.01\right)$, and then rapidly decreases. This decrease occurs when the amount of $\mathrm{CO}_{2}$ in the targets falls below a certain value. For the mixture with water this value is about $30 \%$ (see Figure 1). In the case of pure $\mathrm{CO}_{2}$, ozone decreases when about $50 \%$ of initial $\mathrm{CO}_{2}$ is converted into $\mathrm{CO}$ and other molecules (Figure 6). We have not observed $\mathrm{O}_{3}$ (nor its precursors $\mathrm{O}$, and $\mathrm{O}_{2}$ ) after irradiation of pure water ice although we are confident that some amount of precursors are formed. The lack of detection is partially due to the fact that IR spectroscopy has not the necessary sensitivity to detect "small" amount of $\mathrm{O}_{3}$ that clearly appears only when additional oxygen is available as in the case of the present experiments and others available in the literature [25].

From Figure 6 the relative ratio $\mathrm{O}_{3} / \mathrm{CO}_{2}$ seems to increase again after a given dose $(\sim 9 \mathrm{eV} / 16 \mathrm{u})$, although the majority of the initial $\mathrm{CO}_{2}$ has been consumed. In our opinion this is an artifact of our analysis: the intensities of all of the bands decrease so that the relative error in the evaluation of the band area increases.

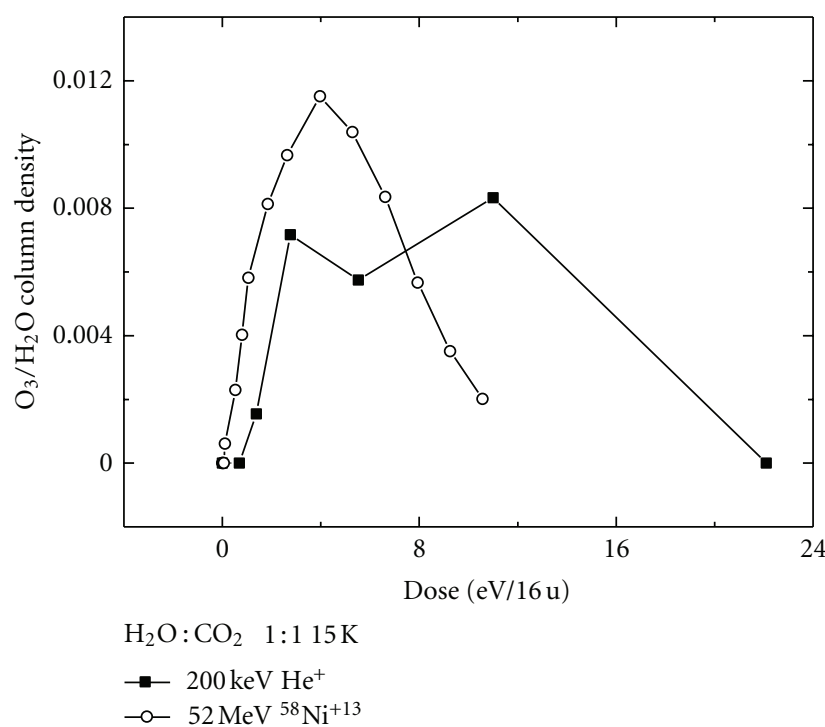

Figure 5: The column density ratio $\left(\mathrm{O}_{3} / \mathrm{H}_{2} \mathrm{O}\right)$ of a mixture $\mathrm{H}_{2} \mathrm{O}: \mathrm{CO}_{2}=1: 1$ are shown versus the dose adsorbed by irradiation with $200 \mathrm{keV} \mathrm{He}^{+}$or $52 \mathrm{MeV} \mathrm{Ni}^{+13}$ at $15 \mathrm{~K}$.

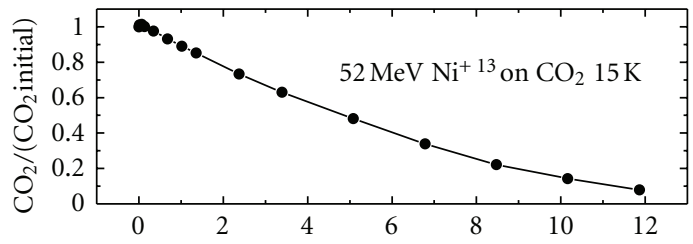

(a)

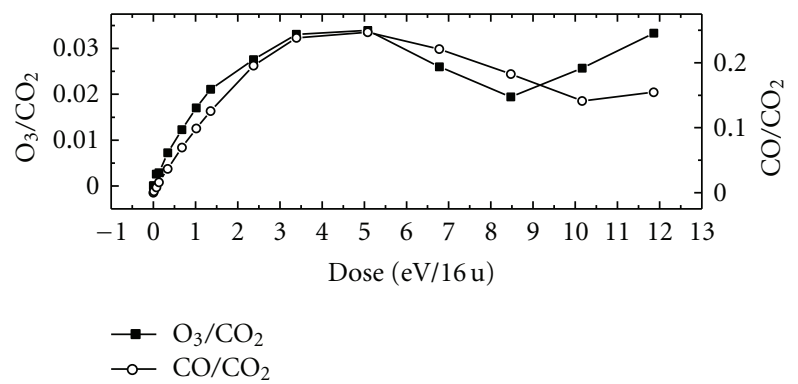

(b)

FIGURE 6: (a) The column density ratio $\left(\mathrm{CO}_{2} / \mathrm{CO}_{2}\right.$ initial $)$ of a pure $\mathrm{CO}_{2}$ target is shown versus the dose adsorbed by irradiation with $52 \mathrm{MeV} \mathrm{Ni}^{+13}$ at $15 \mathrm{~K}$. (b) The column density ratios $\mathrm{CO} / \mathrm{CO}_{2}$ (right scale) and $\mathrm{O}_{3} / \mathrm{CO}_{2}$ (left scale) is shown versus the dose adsorbed by irradiation of a pure $\mathrm{CO}_{2}$ target with $52 \mathrm{MeV} \mathrm{N}^{+13}$ at $15 \mathrm{~K}$.

\section{Discussion}

\subsection{Summary of the Experimental Results}

(i) The $\mathrm{CO}_{2} / \mathrm{H}_{2} \mathrm{O}$ ratio in irradiated $\mathrm{H}_{2} \mathrm{O}: \mathrm{CO}_{2}$ mixtures progressively decreases to a value of $\sim 0.08$.

(ii) $\mathrm{H}_{2} \mathrm{O}_{2}$ has been produced whatever are the projectiles, the temperature, and $\mathrm{H}_{2} \mathrm{O}: \mathrm{CO}_{2}$ mixture. Irradiation of pure water ice produces hydrogen peroxide as well. 
(iii) In pure water ice the $\mathrm{H}_{2} \mathrm{O}_{2} / \mathrm{H}_{2} \mathrm{O}$ number ratio stabilizes to a value of $\sim 0.02$ at low $T$ and of $\sim 0.01$ at higher $T$. As the amount of $\mathrm{CO}_{2}$ in the mixture increases the maximum value of that ratio increases.

(iv) $\mathrm{O}_{3}$ has been detected only after ion irradiation of pure $\mathrm{CO}_{2}$ and $\mathrm{CO}_{2}$ rich mixtures. Its column density increases with ion fluence (up to $\mathrm{O}_{3} / \mathrm{H}_{2} \mathrm{O} \sim 0.01$ ), reaches a maximum, and then decreases.

The results presented in this paper are relevant to understand the chemical evolution of ices in several environments. Water ice, often mixed with other species such as carbon dioxide, is in fact dominant among the constituents of icy mantles of interstellar and circumstellar grains (e.g., [26-28]) and the surfaces of many objects in the Solar System. These latter include Mars whose icy particles in the atmosphere and surface in the polar-subpolar regions are dominated by frozen $\mathrm{CO}_{2}$ [29], comets, Pluto, and other trans-Neptunian objects that are an aggregate of silicates, ices, and complex organic materials. Here we will not discuss those objects but concentrate on the icy moons in the outer Solar System, namely, the Jovian, the Saturnian and the Uranian moons.

4.1.1. The Galilean Jovian Moons. The reflectance spectra of Galilean satellites exhibit differences between the leading and trailing hemispheres that are attributed since a long time [30] to bombardment by energetic charged particles. This is one of a number of space weathering processes [31] that, as said, produces new species in the surface and ejects volatiles that can sustain an atmosphere, or be a source of the local plasma [32]. The surfaces of Europa, Ganimede, and Callisto are dominated by water ice. Io has a surface rich in sulfur-bearing materials continuously ejected by volcanic craters [33]. Some observations by Galileo Orbiter suggest that Europa [34], Ganymede and Callisto [35] could have a subsurface ocean. Thus it is important to know the relationship between the ocean composition and that of the surface and the atmosphere. Cratering studies suggest that average geologic ages of the surfaces are of the order of $10^{7}$ years for Europa, $5 \times 10^{8}$ years for Ganymede, and $4 \times 10^{9}$ years for Callisto [36].

Ion bombardment can alter the optical surface on shorter time scales and produces chemical changes at micron to meter depths in times of $\sim 10$ to $10^{9}$, years respectively [37]. However because of downward mixing by meteorite gardening, sublimation and burial, and subduction, the chemically altered material can be trapped at depths much greater than the radiation penetration depth (e.g., [6]). So oxidants formed at the surface have been suggested as an energy source for possible subsurface biological activity [38].

Water ice is the dominant species on Europa, Callisto, and Ganymede. However IR absorption features observed at $3.4\left(\sim 2940 \mathrm{~cm}^{-1}\right), 3.5\left(\sim 2857 \mathrm{~cm}^{-1}\right), 3.88(\sim$ $\left.2580 \mathrm{~cm}^{-1}\right), 4.05\left(\sim 2470 \mathrm{~cm}^{-1}\right), 4.25\left(\sim 2350 \mathrm{~cm}^{-1}\right)$, and $4.57\left(\sim 2190 \mathrm{~cm}^{-1}\right) \mu \mathrm{m}$ [39] have been attributed to $\mathrm{C}-\mathrm{H}$ groups, $\mathrm{H}_{2} \mathrm{O}_{2}, \mathrm{~S}-\mathrm{H}, \mathrm{SO}_{2}, \mathrm{CO}_{2}$, and $\mathrm{CN}$, respectively. It has been also suggested that $\mathrm{H}_{2} \mathrm{CO}_{3}$ is a more likely candidate for the band at $3.88 \mu \mathrm{m}$ [40]. In addition, abundant surface constituents of the Galilean moons are darker, nonice materials [41], and, in the case of Europa, hydrated sulfuric acid [42]. Some of the darker materials could be organic compounds present at the time of the satellite formation or later delivered by cometary and meteoritic bombardment [6]. Results based on space observations have shown that most of the $\mathrm{CO}_{2}$ detected on the surfaces of Callisto and Ganymede is contained in the nonice materials [43, 44]. Its origin is still controversial: an open question is if it native from the satellite (endogenic) or it is produced by exogenic processes. The exogenic sources might be implantation of carbon ions coming from the Jupiter's magnetosphere or ion-induced synthesis at the interface between water ice and carbonaceous materials eventually left over by meteoritic bombardment [45].

Icy Galilean satellites orbit within Jupiter's giant magnetosphere and their surfaces are subjected to intense bombardment by protons and ions such as $\mathrm{H}^{+}, \mathrm{S}^{n+}$, and $\mathrm{O}^{n+}$ and by energetic electrons [46]. The global flux of energy $\left(\mathrm{keV} \mathrm{cm}^{-2} \mathrm{sec}^{-1}\right)$ due to charged particle (ions and electrons) has been estimated to be $8 \times 10^{10}$ at Europa, 5 $\times 10^{9}$ at Ganymede poles, $2 \times 10^{8}$ at Ganymede equator, and $2 \times 10^{8}$ at Callisto [6]. The energy is primarily carried by energetic particles and not the plasma [47]. As usual we refer to ions and electrons below about $10 \mathrm{keV}$ as "plasma" and above as "energetic particles." The plasma nearly corotates with Jupiter and then it flows preferentially onto the hemisphere trailing the satellite's motion. The less abundant but higher energy particles bombard the satellite in more complex ways [6]. The experiments whose results are here reported are more appropriate to simulate energetic ion bombardment for which electronic dominates over nuclear stopping powers although implantation experiments done with ions and energies having important contribution from nuclear stopping give analogous results [12].

It has been suggested by many groups, and we here confirm, that ion bombardment is the best candidate to explain the presence of hydrogen peroxide on the surfaces of the water-ice-dominated Jovian moons. As an example, assuming that all of the energy is delivered by $200 \mathrm{keV}$ protons, the equilibrium value for the $\mathrm{H}_{2} \mathrm{O}_{2} / \mathrm{H}_{2} \mathrm{O}$ number ratio $(\sim 0.01)$ is obtained in the laboratory after a fluence of about $10^{15}$ ions $\mathrm{cm}^{-2}$, that is, in less than 1 year on Europa and about 30 years on Callisto.

What we want to outline here is that we expect a correlation between the $\mathrm{CO}_{2}$ abundance and the presence of hydrogen peroxide and ozone. By the way please note that the so-called sulfur and carbon cycles determined by ionizing radiations that can both form and destroy carbon or sulfur bearing molecules are intrinsically different ones from the others: the presence of $\mathrm{SO}_{2}$ favors the formation of sulfur polymers (as it occurs with hydrocarbons); the presence of $\mathrm{CO}_{2}$ drives the formation of volatiles (e.g., $\mathrm{CO}$ ) and carbon oxides. Thus most of the sulfur budget is "preserved" on the irradiated moons but most of the carbon that originally forms $\mathrm{CO}_{2}$ is lost.

Future space observations would be able to map the surface composition of the moons (along with the local 
energetic ion fluxes). The measurement of the $\mathrm{CO}_{2} / \mathrm{H}_{2} \mathrm{O}$ number ratio would be correlated to the exposure time and to the local ion flux. A ratio significantly higher than 0.08 would testify for a recent delivery of carbon dioxide at the surface (by external or internal sources) or to an exposure to a low level of energetic ion fluxes. The abundance of hydrogen peroxide and ozone would allow to discriminate between the two possibilities. $\mathrm{H}_{2} \mathrm{O}_{2} / \mathrm{H}_{2} \mathrm{O}$ number ratio significantly higher than $\sim 0.01$ and the presence of ozone would in fact indicate a high level of energetic processing.

4.1.2. The Major Saturnian Moons. The major of the Saturnian moons are usually divided in inner (Mimas, Enceladus, Tethys, and Dione) and outer (Rhea, Titan, Hyperion and Iapetus) with respect to the E-ring. All of these moons, but Titan and Iapetus, have water-ice-rich surfaces [48]. The Saturnian moons and the ring particles exert a strong influence on the magnetosphere of Saturn. The plasma in the magnetosphere corotates with the planet and is implanted in the trailing hemispheres of the moons while energetic particles have a more complex flux distributions. It has been estimated [49] that, at Dione, the global flux of energy $\left(\mathrm{keVcm}^{-2} \mathrm{sec}^{-1}\right)$ is $3 \times 10^{9}$ that, assuming that all of the energy is delivered by $200 \mathrm{keV}$ protons, correspond to a time of less than two years to reach the equilibrium value for the $\mathrm{H}_{2} \mathrm{O}_{2} / \mathrm{H}_{2} \mathrm{O}$ number ratio $(\sim 0.01)$. At present hydrogen peroxide has not been detected, although it is easy to predict it should be present and in fact it has been suggested that it could be responsible for the ultraviolet spectral slopes in the reflectance of the Saturnian moons [50]. Ozone has been observed on Dione and Rhea [5] and its presence has been attributed to radiolysis of pure water in the hypothesis that $\mathrm{O}_{2}$ is formed first. In fact $\mathrm{O}_{2}$ has been detected to form a tenuous atmosphere around the moons [51]. However, at present time, there is no experimental evidence of the formation of appreciable quantity of ozone after irradiation of pure water ice. Ozone has been produced only after addition in the ice of other O-bearing species such as $\mathrm{O}_{2}$ [25] and $\mathrm{CO}_{2}$ as discussed in this paper. Because at the temperatures of the surfaces of the moons $(50-100 \mathrm{~K})$, it is difficult to trap $\mathrm{O}_{2}$; we believe that $\mathrm{CO}_{2}$ is a better candidate as parent molecule of the observed ozone.

Particularly attractive in recent years has been the study of Enceladus, the smallest geologically active body in the Solar System [46]. Its surface is highly variegated and exhibits both ancient (crater rich) and young areas. The south pole area is warm and give rise to a large plume (more than $100 \mathrm{~kg} / \mathrm{s}$ are released) which is the main source of ions in the magnetosphere of Saturn. There are many molecules ejected in the gas phase such as water vapor, carbon dioxide, and nitrogen (for a complete list see Table 1 in [52]). These molecules are likely incorporated in the ice where solid $\mathrm{CO}_{2}$ and organics have been detected [53]. It has been suggested that Enceladus may have liquid water underneath the south polar surface where, analogously to Europa, biological activity might be developed. In addition the presence of cracks would favour the exchange of materials from the surface to the (liquid?) interiors [52]. Thus oxidants formed at the surface would be able to furnish chemical energy for biological activities. At present, however, there is no consensus on the presence of hydrogen peroxide on the surface of Enceladus, an IR band has been observed at about $2830 \mathrm{~cm}^{-1}$. The attribution of this band to hydrogen peroxide [54] has been questioned [55]; presently it is attributed to short chain carbons [53] or to methanol [56].

Of the two major Saturnian moons whose surfaces are not dominated by water ice, Titan, because of its atmosphere (surface pressure of $1.5 \mathrm{~atm}$ ), has a surface that is preserved from the irradiation of energetic ions and is out from the interest of the present paper.

Iapetus has unusual surface characteristic: its leading hemisphere is dark and its trailing hemisphere is almost as bright as ice. The origin and composition of the dark material is still object of debate. Instruments on board of the CassiniHuygens space vehicle detected frozen $\mathrm{CO}_{2}$ on the dark side by the observation of the band at about $2343 \mathrm{~cm}^{-1}$ [57]. The high loss/destruction rate of $\mathrm{CO}_{2}$ predicted at the Iapetus temperatures requires an active source. It has been suggested that photolysis of mixtures of water ice with carbonaceous solids is the primary mechanism [58]. Iapetus orbits outside of Saturn's magnetosphere and the ions interacting with Iapetus' surface are from the solar wind and from cosmic ions. Assuming that the flux of 2 ions $\mathrm{cm}^{-2} \mathrm{sec}^{-1}$ measured for cosmic ions [49] is well represented by our experiments done by using $58 \mathrm{MeV} \mathrm{Ni}$, the equilibrium value of $\sim 0.01$ in the $\mathrm{H}_{2} \mathrm{O}_{2} / \mathrm{H}_{2} \mathrm{O}$ number ratio is obtained in about $2 \times 10^{4} \mathrm{yrs}$. The dark emisphere could accumulate larger quantities of hydrogen peroxide in view of the presence of $\mathrm{CO}_{2}$.

4.1.3. The Major Uranian Moons. Uranus has five major moons: Miranda, Ariel, Umbriel, Titania, and Oberon. Miranda is dominated by water ice, while the other moons are made by approximately equal amounts of rock and ice. Their relatively dark colours suggest the presence of carbonaceous material possibly coming from the uranian ring. It has been suggested that Titania and Oberon may harbor liquid water oceans at the core/mantle interface [59]. Among the "contaminants" present on the surface of the moons, $\mathrm{CO}_{2}$ has been discovered first on Ariel [60], opening interesting question on its origin (exogenic versus endogenic). Its presence and surface distribution on Umbriel and Titania (and its absence on Oberon) has been studied by Grundy et al. [61]. They found that the strength of the observed IR bands decreases with the distance from the planets and is greater on the trailing hemispheres. This is not inconsistent with an endogenic origin governed by plasma ion bombardment although alternative possibilities exist. As said the experiments here discussed are more appropriate to simulate energetic ion bombardment whose fluxes and complex distribution on the surfaces of the Uranian moons are not known. It is, however, easily predictable that some hydrogen peroxide has to be present everywhere along with some patches of ozone transiently formed where $\mathrm{CO}_{2}$ is more abundant and freshly exposed. 


\subsection{Summary of Observations, Suggestions, and Conclusion}

\subsubsection{Observations.}

(i) Hydrogen peroxide has been found on the surface of Europa, Ganymede, and Callisto [2, 3].

(ii) Ozone has been observed on some Galilean satellites [4] and on Saturn's satellites Rhea and Dione [5].

(iii) Oxidants formed at the surface have been suggested as an energy source for possible subsurface biological activity [38].

(iv) Icy moons in the outer Solar System are subjected to intense fluxes of energetic ions that alter the optical surface and produces chemical changes at micron to meter depths in times of $\sim 10$ to $10^{9}$ years, respectively $[6,37]$.

\subsubsection{Suggestions.}

(i) Ion irradiation is the primary candidate to explain the presence of $\mathrm{H}_{2} \mathrm{O}_{2}$ on icy moons.

(ii) Ozone could be produced by ion irradiation. It would be correlated with $\mathrm{CO}_{2}$ and should be searched for whenever $\mathrm{CO}_{2}$ is observed.

(iii) Oxidants formed at the surface by ion irradiation could diffuse and reach the putative subsurface ocean. They would then be able to sustain biological activities.

4.2.3. Conclusion. It appears to be important, although not easy, to plan experiments in which $\mathrm{CO}_{2}$ is synthesized by ion irradiation (at plasma energies and above) of water ice mixed or deposited on carbonaceous solids while looking also to the oxidant formation. This would allow to clarify if carbon dioxide can be efficiently formed by exogenic processes (ion bombardment) to an abundance high enough to induce the production of ozone and other oxidants. On the other side observations that maps at the highest possible spatial resolution the composition of the surfaces are necessary to better understand the physico-chemical processes that govern the evolution of the cold worlds where liquid subsurface oceans could harbor biological activities.

\section{Acknowledgment}

This research has been supported by the European COST Action CM0805: The Chemical Cosmos.

\section{References}

[1] B. Schmitt, C. de Bergh, and M. Festou, Solar System Ices, Kluwer Academic Publishers, Dordrecht, The Netherlands, 1998.

[2] R. W. Carlson, M. S. Anderson, R. E. Johnson et al., "Hydrogen peroxide on the surface of Europa," Science, vol. 283, no. 5410, pp. 2062-2064, 1999.

[3] A. R. Hendrix, C. A. Barth, and C. W. Hord, "Ganymede's ozone-like absorber: observations by the Galileo ultraviolet spectrometer," Journal of Geophysical Research, vol. 104, no. 6, pp. 14169-14178, 1999.

[4] K. S. Noll, R. E. Johnson, A. L. Lane, D. L. Domingue, and H. A. Weaver, "Detection of ozone on Ganymede," Science, vol. 273, no. 5273, pp. 341-343, 1996.

[5] K. S. Noll, T. L. Roush, D. P. Cruikshank, R. E. Johnson, and Y. J. Pendleton, "Detection of ozone on Saturn's satellites Rhea and Dione," Nature, vol. 388, pp. 45-47, 1997.

[6] R. E. Johnson, R. W. Carlson, J. F. Cooper, C. Paranicas, M. H. Moore, and M. C. Wong, "Radiation effects on the surfaces of the Galilean satellites," in Jupiter: Planet, Satellites and Magnetosphere, F. Bagenal, W. B. McKinnon, and T. E. Dowling, Eds., vol. 1, pp. 485-512, Cambridge University Press, Cambridge, UK, 2004.

[7] W. L. Brown, L. J. Lanzerotti, J. M. Poate, and W. M. Augustyniak, "Sputtering of ice by MeV ions," Physical Review Letters, vol. 49, pp. 1027-1030, 1978.

[8] R. A. Baragiola, "Energetic electronic processes on extraterrestrial surfaces," Nuclear Instruments and Methods in Physics Research, vol. 232, no. 1-4, pp. 98-107, 2005.

[9] R. E. Johnson, M. Famá, M. Liu, R. A. Baragiola, E. C. Sittler Jr., and H. T. Smithd, "Sputtering of ice grains and icy satellites in Saturn's inner magnetosphere," Planetary and Space Science, vol. 56, no. 9, pp. 1238-1243, 2008.

[10] E. Seperuelo Duarte, P. Boduch, H. Rothard et al., "Heavy ion irradiation of condensed $\mathrm{CO}_{2}$ sputtering and molecule formation," Astronomy \& Astrophysics, vol. 502, no. 2, pp. 599603, 2009.

[11] E. Seperuelo Duarte, A. Domaracka, P. Boduch, H. Rothard, E. Dartois, and E. F. da Silveira, "Laboratory simulation of heavyion cosmic-ray interaction with condensed CO," Astronomy \& Astrophysics, vol. 512, article A71, 2010.

[12] O. Gomis, M. A. Satorre, G. Strazzulla, and G. Letob, "Hydrogen peroxide formation by ion implantation in water ice and its relevance to the Galilean satellites," Planetary and Space Science, vol. 52, no. 5-6, pp. 371-378, 2004.

[13] G. Strazzulla, G. Leto, F. Spinella, and O. Gomis, "Production of oxidants by ion irradiation of Water/Carbon dioxide frozen mixtures," Astrobiology, vol. 5, no. 5, pp. 612-621, 2005.

[14] S. Pilling, E. Seperuelo Duarte, A. Domaracka, H. Rothard, P. Boduch, and E. F. da Silveira, "Radiolysis of $\mathrm{H}_{2} \mathrm{O}: \mathrm{CO}_{2}$ ices by heavy energetic cosmic ray analogs," Astronomy \& Astrophysics, vol. 523, article A77, 12 pages, 2010.

[15] G. Strazzulla, G. A. Baratta, and M. E. Palumbo, "Vibrational spectroscopy of ion-irradiated ices," Spectrochim Acta, vol. 57, no. 4, pp. 825-842, 2001.

[16] J. F. Ziegler, J. P. Biersack, and M. D. Ziegler, The Stopping and Range of Ions in Solids, Pergamon Press, New York, NY, USA, 2008.

[17] G. Strazzulla and R. E. Johnson, "Irradiation effects on comets and cometary debris," in Comets in The Post-Halley Era, R. L. Newburn Jr., M. Neugebauer, and J. Rahe, Eds., vol. 1, pp. 243275, Kluwer Academic Publishers, London, UK, 1991.

[18] L. J. Allamandola, S. A. Sandford, and G. J. Valero, "Photochemical and thermal evolution of interstellar/precometary ice analogs," Icarus, vol. 76, no. 2, pp. 225-252, 1988.

[19] H. Yamada and W. B. Person, "Absolute infrared intensities of the fundamental absorption bands in solid $\mathrm{CO}_{2}$ and $\mathrm{N}_{2} \mathrm{O}$," Journal of Chemical Physics, vol. 41, no. 8, 10 pages, 1964.

[20] M. H. Moore and R. L. Hudson, "IR detection of $\mathrm{H}_{2} \mathrm{O}_{2}$ at $80 \mathrm{~K}$ in ion-irradiated laboratory ices relevant to Europa," Icarus, vol. 145, no. 1, pp. 282-288, 2000.

[21] M. A. H. Smith, C. P. Rinsland, V. M. Devi, L. S. Rothman, and K. N. Rao, "Intensities and collision broadening parameters 
from infrared spectra," in Molecular Spectroscopy, K. N. Rao, Ed., vol. 3, pp. 112-248, Academic Press, New York, NY, USA, 1985.

[22] M. E. Palumbo, "Formation of compact solid water after ion irradiation at $15 \mathrm{~K}$," Astronomy \& Astrophysics, vol. 453, no. 3, pp. 903-909, 2006.

[23] L. S. Farenzena, V. M. Collado, and E. F. D. A. Silveira, "Secondary Ion emission from $\mathrm{CO}_{2}-\mathrm{H}_{2} \mathrm{O}$ ice irradiated by energetic heavy ions," International Journal of Mass Spectrometry, vol. 243, no. 1, pp. 85-93, 2005.

[24] C. R. Ponciano, L. S. Farenzena, V. M. Collado, E. F. da Silveira, and K. Wien, "Secondary ion emission from $\mathrm{CO}_{2}-\mathrm{H}_{2} \mathrm{O}$ ice irradiated by energetic heavy ions: part II: analysis—-search for organic molecular ions," International Journal of Mass Spectrometry, vol. 244, no. 1, pp. 41-49, 2005.

[25] P. D. Cooper, M. H. Moore, and R. L. Hudson, "Radiation chemistry of $\mathrm{H}_{2} \mathrm{O}+\mathrm{O}_{2}$ ices," Icarus, vol. 194, no. 1, pp. 379$388,2008$.

[26] S. P. Willner, F. C. Gillett, T. L. Herter et al., "Infrared spectra of protostars-composition of the dust shells," Astrophysical Journal, vol. 253, pp. 174-187, 1982.

[27] P. A. Gerakines, D. C. B. Whittet, P. Ehrenfreund et al., "Observations of solid carbon dioxide in molecular clouds with the infrared space observatory," Astrophysical Journal, vol. 522, no. 1, pp. 357-377, 1999.

[28] K. M. Pontoppidan, A. C. A. Boogert, H. J. Fraser et al., "The c2d spitzer spectroscopic survey of ices around low-mass Young Stellar objects. II. $\mathrm{CO}_{2}$," Astrophysical Journal, vol. 678, no. 2, pp. 1005-1031, 2008.

[29] J.-P. Bibring, Y. Langevin, F. Poulet et al., "Perennial water ice identified in the south polar cap of Mars," Nature, vol. 428, pp. 627-630, 2004.

[30] D. Morrison, Satellites of Jupiter, University of Arizona Press, Tucson, Ariz, USA, 1982.

[31] B. E. Clark and R. E. Johnson, "Interplanetary weathering: surface erosion in outer space," Eos, Transactions, American Geophysical Union, vol. 77, no. 15, pp. 141-145, 1996.

[32] R. E. Johnson, "Sputtering and desorption from Icy surfaces," in Solar System Ices, B. Schmitt and C. deBergh, Eds., vol. 227, pp. 303-334, Kluwer Academic Publishers, Dodrecht, The Netherlands, 1998.

[33] J. S. Kargel, J. Z. Kaye, J. W. Head III et al., "Europa's crust and ocean: origin, composition, and the prospects for life," Icarus, vol. 148, no. 1, pp. 226-265, 2000.

[34] K. K. Khurana, M. G. Kivelson, D. J. Stevenson et al., "Induced magnetic fields as evidence for subsurface oceans in Europa and Callisto," Nature, vol. 395, pp. 777-780, 1998.

[35] C. Zimmer, K. Khurana, and M. G. Kivelson, "Subsurface oceans on Europa and Callisto: constraints from Galileo magnetometer observations," Icarus, vol. 147, no. 2, pp. 329$347,2000$.

[36] K. Zahnle, L. Dones, and H. F. Levison, "Cratering rates on the Galilean satellites,” Icarus, vol. 136, no. 2, pp. 202-222, 1998.

[37] J. F. Coope, R. E. Johnson, B. H. Mauk, H. B. Garret, and N. Gehrels, "Energetic ion and electron irradiation of the icy Galilean satellites," Icarus, vol. 149, no. 1, pp. 133-159, 2001.

[38] C. F. Chyba and K. P. Hand, "Planetary science life without photosynthesis,” Science, vol. 292, no. 5524, pp. 2026-2027, 2001.

[39] T. B. McCord, R. W. Carlson, W. D. Smythe et al., "Organics and other molecules in the surfaces of Callisto and Ganymede," Science, vol. 278, no. 5336, pp. 271-275, 1997.
[40] W. Hage, K. R. Liedl, A. Hallbrucker, and E. Mayer, "Carbonic acid in the gas phase and its astrophysical relevance," Science, vol. 279, no. 5355, pp. 1332-1335, 1998.

[41] T. B. McCord, G. B. Hansen, R. N. Clark et al., "Nonwater-ice constituents in the surface material of the icy Galilean satellites from the Galileo near-infrared mapping spectrometer investigation "' Geophysical Research, vol. 103, pp. 8603-8626, 1998.

[42] R. W. Carlson, R. E. Johnson, and M. S. Anderson, "Sulfuric acid on Europa and the radiolytic sulfur cycle," Science, vol. 286, no. 5437, pp. 97-99, 1999.

[43] C. A. Hibbitts, J. E. Klemaszewski, T. B. McCord, G. B. Hansen, and R. Greeley, "CO 2 -rich impact craters on Callisto," Journal of Geophysical Research-Planets, vol. 107, article 5084, 2002.

[44] C. A. Hibbitts, R. T. Pappalardo, G. B. Hansen, and T. B. McCord, "Carbon dioxide on ganymede," Journal of Geophysical Research, vol. 108, article 5036, 22 pages, 2003.

[45] G. Strazzulla, "Cosmic ion bombardment of the icy moons of Jupiter," Nuclear Instruments and Methods in Physics Research Section B, vol. 269, no. 9, pp. 842-851, 2011.

[46] C. C. Porco, P. Helfenstein, P. C. Thomas et al., "Cassini observes the active south pole of Enceladus," Science, vol. 311, no. 5766, pp. 1393-1401, 2006.

[47] B. H. Mauk, S. A. Gary, M. Kane, E. P. Keath, S. M. Krimigis, and T. P. Armstrong, "Hot plasma parameters of Jupiter's inner magnetosphere," Geophysical Research, vol. 101, no. 4, pp. 7685-7695, 1996.

[48] P. C. Thomas, J. A. Burns, P. Helfenstein et al., "Shapes of the saturnian icy satellites and their significance," Icarus, vol. 190, no. 2, pp. 573-584, 2007.

[49] T. Madeya, R. E. Johnson, and T. Orlando, "Far-out surface science: radiation-induced surface processes in the solar system," Surface Science, vol. 500, no. 1-3, pp. 838-858, 2002.

[50] B. H. Mauk, D. C. Hamilton, T. W. Hill et al., "Fundamental plasma processes in Saturn's Magnetosphere," in Saturn from Cassini-Huygens, M. K. Dougherty, L. W. Esposito, and S. M. Krimigis, Eds., pp. 281-331, Springer, Amsterdam, The Netherlands, 2009.

[51] R. E. Johnson, J. G. Luhmann, and R. L. Tokar, "Production, ionization and redistribution of $\mathrm{O}_{2}$ in Saturn's ring atmosphere," Icarus, vol. 180, no. 2, pp. 393-402, 2008.

[52] C. D. Parkinson, M.-C. Liang, H. Hartman et al., "Enceladus: cassini observations and implications for the search for life," Astronomy \& Astrophysics, vol. 463, no. 1, pp. 353-357, 2007.

[53] R. H. Brown, R. N. Clark, B. J. Buratti et al., "Composition and physical properties of Enceladus' surface," Science, vol. 311, no. 5766, pp. 1425-1428, 2006.

[54] S. F. Newman, B. J. Buratti, and R. Jaumann, "Hydrogen peroxide on Enceladus," Astrophysical Journal, vol. 670, no. 2, pp. L143-L146, 2007.

[55] M. J. Loeffler and R. A. Baragiola, "Is the $3.5 \mu \mathrm{m}$ infrared feature on Enceladus due to hydrogen peroxide?" Astrophysical Journal Letters, vol. 694, no. 1, pp. L92-L94, 2009.

[56] R. Hodyss, C. D. Parkinson, P. V. Johnson et al., "Methanol on enceladus," Geophysical Research Letters, vol. 36, Article ID L17103, 3 pages, 2009.

[57] B. J. Buratti, D. P. Cruikshank, R. H. Brown et al., "Cassini visual and infrared mapping spectrometer observations of Iapetus: detection of $\mathrm{CO}_{2}$," Astrophysical Journal, vol. 622, no. 2, pp. L149-L152, 2005.

[58] E. E. Palmer and R. H. Brown, "Production and detection of carbon dioxide on Iapetus," Icarus, vol. 212, no. 2, pp. 807$818,2011$. 
[59] H. Hussmann, F. Sohl, and T. Spohn, "Subsurface oceans and deep interiors of medium-sized outer planet satellites and large trans-neptunian objects," Icarus, vol. 185, no. 1, pp. 258273, 2006.

[60] W. M. Grundy, L. A. Young, and E. F. Young, "Discovery of $\mathrm{CO}_{2}$ ice and leading-trailing spectral asymmetry on the uranian satellite Ariel," Icarus, vol. 162, pp. 223-230, 2003.

[61] W. M. Grundy, L. A. Youngb, J. R. Spencer, R. E. Johnso, E. F. Young, and M. W. Buie, "Distributions of $\mathrm{H}_{2} \mathrm{O}$ and $\mathrm{CO}_{2}$ ices on Ariel, Umbriel, Titania, and Oberon from IRTF/SpeX observations," Icarus, vol. 184, no. 2, pp. 543-555, 2006. 

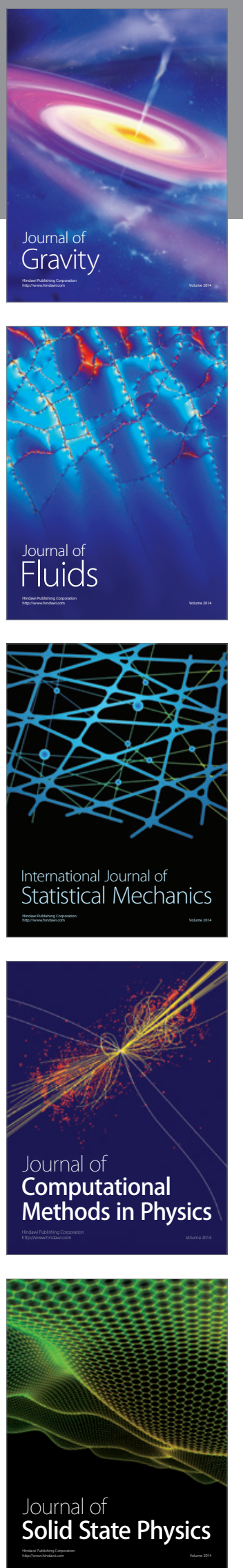

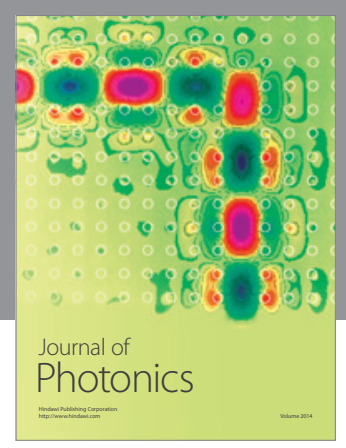

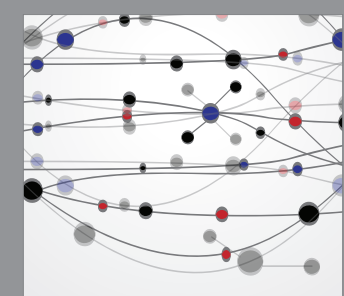

The Scientific World Journal
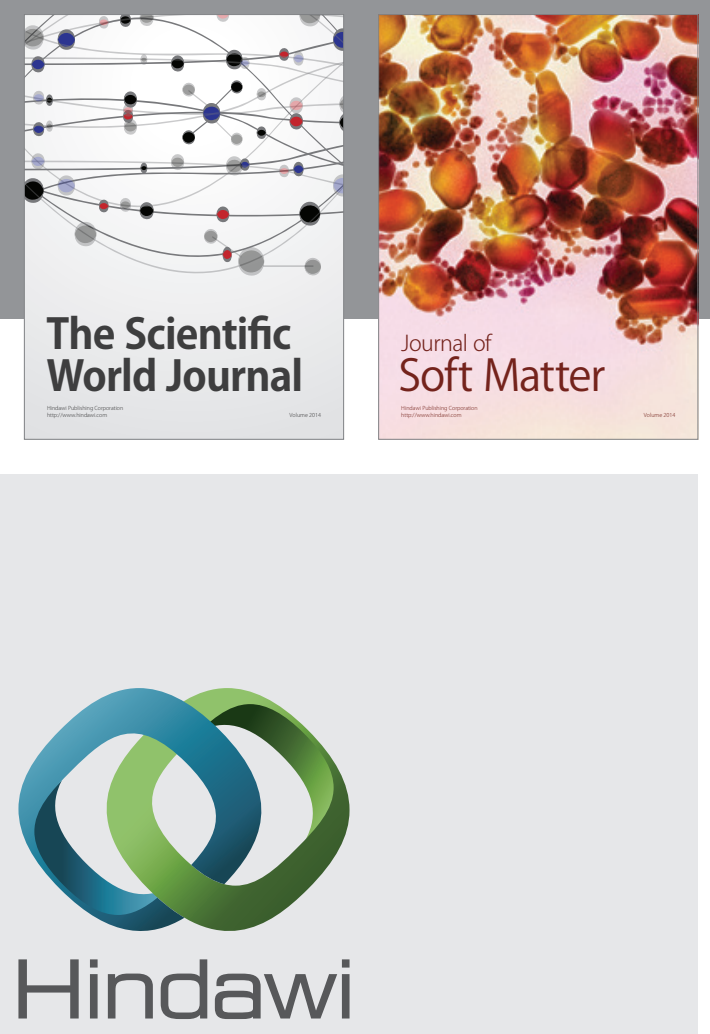

Submit your manuscripts at

http://www.hindawi.com
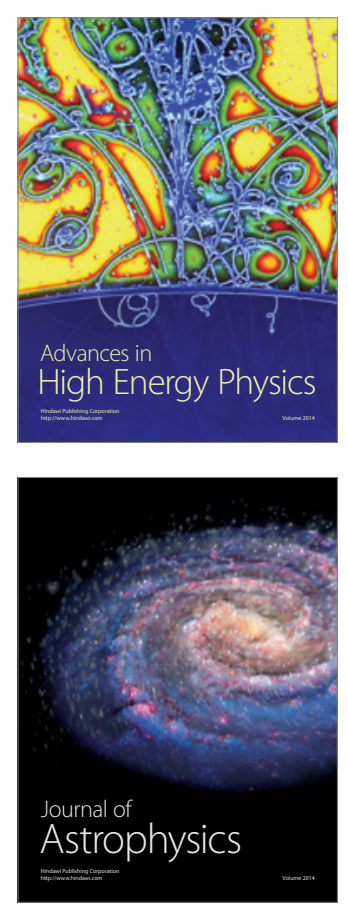
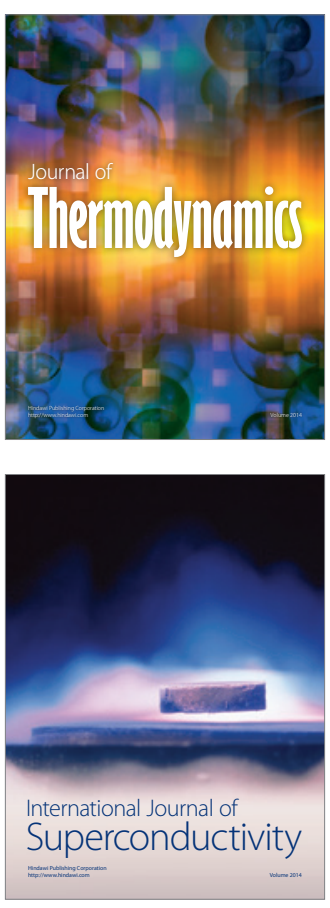
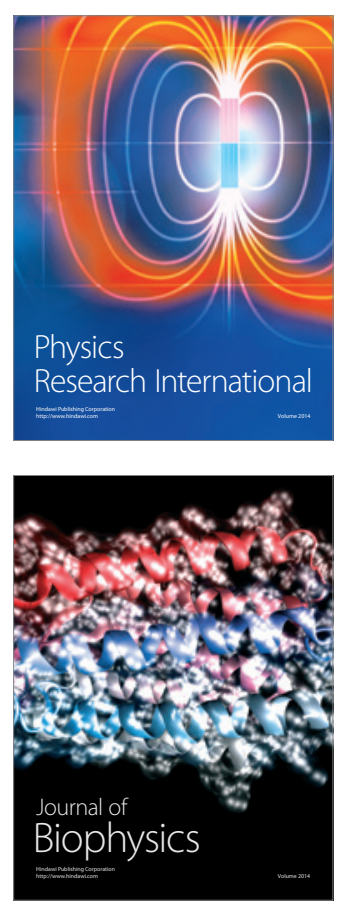
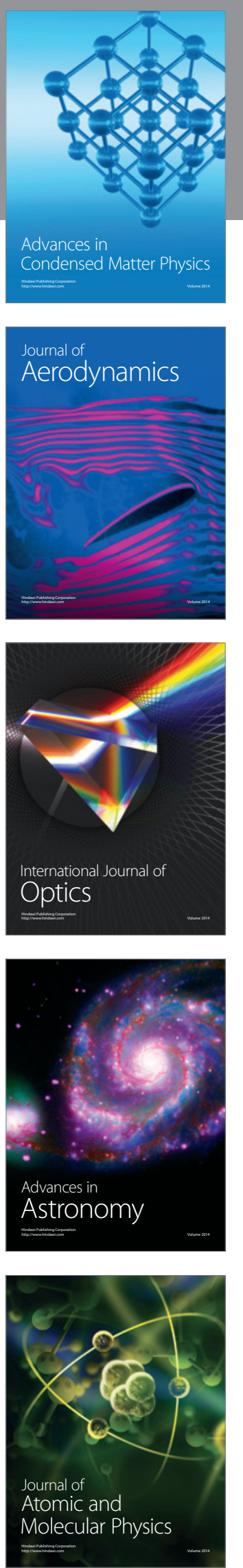